hep-th/9503020

BERC-94/102

March, 1995

BOW-PH-104

BRX-TH-366

\title{
Large N Universality of the Two-Dimensional Yang-Mills String
}

\author{
Michael Crescimanno $\$ \dagger$ \\ Department of Physics \\ Berea College \\ Berea, KY 40404 \\ Stephen G. NACUlich ${ }^{\star}$ \\ Department of Physics \\ Bowdoin College \\ Brunswick, ME 04011 \\ and \\ Howard J. Schnitzer ${ }^{\$ \ddagger}$ \\ Department of Physics \\ Brandeis University \\ Waltham, MA 02254
}

\begin{abstract}
We exhibit the gauge-group independence ("universality") of all normalized non-intersecting Wilson loop expectation values in the large $N$ limit of two-dimensional Yang-Mills theory. This universality is most easily understood via the string theory reformulation of these gauge theories. By constructing an isomorphism between the string maps contributing to normalized Wilson loop expectation values in the different theories, we prove the large $N$ universality of these observables on any surface. The string calculation of the Wilson loop expectation value on the sphere also leads to an indication of the large $N$ phase transition separating strongand weak-coupling phases.
\end{abstract}

\footnotetext{
\$ Research supported in part by the DOE under grant DE-FG02-92ER40706

$\dagger$ crescimanno@berea.edu

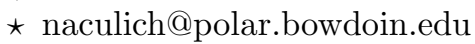

$\ddagger$ schnitzer@binah.cc.brandeis.edu
} 


\section{Introduction}

Pure Yang-Mills theory on an arbitrary (Euclidean) two-manifold is an exactly solvable quantum field theory [1-3]. It has been shown that these theories are string theories [4-10], although generalizing this discovery to gauge theory in higher dimensions has proven elusive.

Wilson loop vacuum expectation values (VEVs) form a complete basis of gaugeinvariant observables of the pure gauge theory. An important class of these observables, the VEVs of non-intersecting Wilson loops, can be written as exact expressions that depend on the representation theory of the gauge group $G$ [3]. For the Lie groups $G=\mathrm{SU}(N), \mathrm{U}(N), \mathrm{SO}(N)$, or $\mathrm{Sp}(N)$, one may expand the Wilson loop observables in $1 / N$ about $N=\infty$; the large $N$, strong-coupling limit is the natural one for making explicit the connection between gauge theory and string theory.

When one computes simple Wilson loops, one discovers that the normalized VEV is independent, in the large $N$ limit, of the choice of gauge group $G$ (e.g., see eqs. (2.3), (2.4), and (2.5) below). This suggests that there is but one theory at large $N$ in two dimensions. At first sight, this conclusion may strike one as either incorrect, or else trivial. We show below that it is correct; here we describe why it is non-trivial. First, in two-dimensional Yang-Mills theory at weak coupling, the form of the gauge field propagators combined with the Feynman diagrams in leading order in $1 / N$ suggest that the computation of any perturbative gaugeinvariant quantity must be the same for all groups at large $N$. What is claimed here and in ref. [11], however, is the universality of observables for all coupling. The third order phase transition separating the strong- and weak-coupling regions of the theory on a two-sphere [12] complicates extending the perturbative equivalence to equivalence at all coupling. Second, the exact gauge theory expressions for Wilson loop VEVs appear to depend on many details of the representation theory of the group $G$, and this large $N$ equivalence requires highly non-trivial cancellations of sub-leading (in $1 / N$ ) terms coming from the dimensions and quadratic Casimirs of 
representations. From the gauge theory point-of-view, large $N$ universality seems miraculous.

Large $N$ gauge-group independence was first noticed in ref. [11], where the large $N$ saddle point on the sphere (and projective plane) was computed for various gauge groups. There it was shown that the saddle point is universal, and the computation of the partition function and non-intersecting Wilson loop for the fundamental representation led to the same result for all groups $G$ at large $N$. From the point of view of this matrix-model-like, large $N$ technique, universality is the result of one's ability to rewrite the expressions for the free energy and Wilson loop on the sphere in a universal form. This does not reveal any deeper reason for the universality.

In this paper, we demonstrate that the string theory reformulation of twodimensional Yang-Mills theory provides the natural framework for understanding large $N$ universality. We show that the large $N$ equivalence of Wilson loop VEVs follows from an isomorphism between string maps in the various theories. We prove an exact (to all orders in the double expansion in the areas and the exponential of the areas) equality of normalized VEVs of non-intersecting Wilson loops in an arbitrary representation on an arbitrary surface for all the classical Lie groups to leading order in $1 / N$. This is a significant demonstration of the utility of the string theory reformulation of two-dimensional Yang-Mills theory.

\section{Wilson loop universality}

Consider a homologically trivial Wilson loop that divides an orientable surface $\mathcal{S}$ of genus $h$ and (dimensionless) area $A$ into a disk $D$ with area $A_{1}$ and a surface $\mathcal{S} \backslash D$ with area $A_{2}$. The expectation value of the trace of this Wilson loop in some representation $R$ of the gauge group $G$ is $[1-3]$

$$
W_{R}=\sum_{R_{1}, R_{2}} N_{G}\left(R_{1}, R ; R_{2}\right)\left(\operatorname{dim} R_{1}\right)\left(\operatorname{dim} R_{2}\right)^{1-2 h} \exp \left[-\frac{A_{1} C_{2}\left(R_{1}\right)+A_{2} C_{2}\left(R_{2}\right)}{2 N f}\right]
$$


where $C_{2}(R)$ and $\operatorname{dim} R$ denote the quadratic Casimir and dimension of the representation $R, N_{G}\left(R_{1}, R ; R_{2}\right)$ are tensor product multiplicities of the group $G$, and $f$ is $\frac{1}{2}$ for $\operatorname{Sp}(N)$ and 1 otherwise. In the string theories corresponding to these Yang-Mills theories, the natural observables are not $W_{R}$ but rather the linear combinations $[6,8,9]$

$$
W_{\kappa}=\sum_{R \in Y_{r}} \chi_{R}(\kappa) W_{R}
$$

where $\kappa$ denotes a conjugacy class of the symmetric group $S_{r}, Y_{r}$ denotes the set of Young diagrams with $r$ cells, and $\chi_{R}(\kappa)$ is the character of the conjugacy class $\kappa$ in the representation $R$ of $S_{r}$. The $W_{\kappa}$ basis, not the irreducible representation basis $W_{R}$, is the appropriate basis in which to compare observables between different gauge theories.

We claim that the large $N$ limit of the normalized VEV $\widehat{W}_{\kappa}=W_{\kappa} / Z$ for nonintersecting Wilson loops (on any surface) is independent of the gauge group. First, we give several examples. Consider the homologically trivial Wilson loop on the sphere with $\kappa=(1)$, a single cycle of length one. Irrespective of the gauge group, the first few terms of its normalized VEV are ${ }^{\star \dagger}$

$$
\begin{aligned}
\widehat{W}_{(1)}=\frac{W_{\mathrm{a}}}{Z} & \underset{N \rightarrow \infty}{\longrightarrow} N\left[\mathrm{e}^{-\frac{1}{2} A_{1}}+\left(\frac{1}{2} A_{1}^{2}-A_{1}-1\right) \mathrm{e}^{-A_{1}-\frac{1}{2} A_{2}}\right. \\
& +\left(\frac{1}{2} A_{1}^{4}+\frac{2}{3} A_{1}^{3} A_{2}+\frac{1}{4} A_{1}^{2} A_{2}^{2}+\frac{8}{3} A_{1}^{3}-\frac{5}{2} A_{1}^{2} A_{2}-\frac{1}{2} A_{1} A_{2}^{2}+\frac{3}{2} A_{1}^{2}\right. \\
& \left.\left.+A_{1} A_{2}-\frac{1}{2} A_{2}^{2}+A_{1}+A_{2}+1\right) \mathrm{e}^{-\frac{3}{2} A_{1}-A_{2}}+\cdots\right]+\left(A_{1} \leftrightarrow A_{2}\right)
\end{aligned}
$$

Likewise, for $\kappa=(2)$, a single cycle of length two,

$$
\begin{aligned}
\widehat{W}_{(2)} & =\frac{W_{\text {品 }}-W_{\text {日 }}}{Z} \\
& \underset{N \rightarrow \infty}{\longrightarrow} N\left[\left(1-A_{1}\right) \mathrm{e}^{-A_{1}}+\left(-\frac{4}{3} A_{1}^{3}+4 A_{1}^{2}\right) \mathrm{e}^{-\frac{3}{2} A_{1}-\frac{1}{2} A_{2}}+\cdots\right]+\left(A_{1} \leftrightarrow A_{2}\right)
\end{aligned}
$$

\footnotetext{
$\star$ Expressions of this type were first given in ref. [2].

$\dagger$ This corrects an error in ref. [13]. See appendix of ref. [11].
} 
The Wilson loop with $\kappa=\left(1^{2}\right)$ has VEV

$$
\begin{aligned}
\widehat{W}_{\left(1^{2}\right)}=\frac{W_{\text {口 }}+W_{\text {日 }}}{Z} \underset{N \rightarrow \infty}{\longrightarrow} N^{2}\left[\mathrm{e}^{-A_{1}}+\mathrm{e}^{-\frac{1}{2} A_{1}-\frac{1}{2} A_{2}}+\left(A_{1}^{2}-2 A_{1}-2\right) \mathrm{e}^{-\frac{3}{2} A_{1}-\frac{1}{2} A_{2}}\right. \\
\left.+\left(\frac{1}{2} A_{1}^{2}+\frac{1}{2} A_{2}^{2}-A_{1}-A_{2}-2\right) \mathrm{e}^{-A_{1}-A_{2}}+\cdots\right]+\left(A_{1} \leftrightarrow A_{2}\right)
\end{aligned}
$$

Observe that $\widehat{W}_{\left(1^{2}\right)}=\left[\widehat{W}_{(1)}\right]^{2}$ in the large $N$ limit; we will see that this has a natural explanation in string theory. This large $N$ factorization is also expected from the existence of the large $N$ saddle point (closely related to the master field) but is only valid for representations whose diagrams have a finite number of cells. At the end of section 3, we give examples of the universal large $N$ limits of Wilson loops (both homologically trivial and non-trivial) on surfaces other than the sphere.

The large $N$ universality of the normalized Wilson loop VEVs exhibited above begs a deeper explanation. The saddle point techniques [12-17] used in ref. [11] give an analytical view of this universality for simple cases, but this approach somewhat obscures the physical grounds for the equivalence. It is more enlightening to describe universality as an equivalence between string theories, to which we now turn.

\section{String theory explanation}

To make the translation from gauge theory to string theory, recall that the features of the string maps are determined by the form of the quadratic Casimir and the dimension of the representations in eq. (2.1). For the classical Lie groups, the quadratic Casimir for tensor representations is

$$
C_{2}(R)=f N\left[r+\frac{T(R)}{N}-U(r)\right]
$$


where

$$
\begin{aligned}
& f= \begin{cases}1 & \text { for } \mathrm{SU}(N), \mathrm{U}(N), \text { and } \mathrm{SO}(N), \\
\frac{1}{2} & \text { for } \operatorname{Sp}(N),\end{cases} \\
& T(R)=\sum_{i=1}^{\text {rank } G} \ell_{i}\left(\ell_{i}+1-2 i\right)=\sum_{i=1}^{k_{1}} \ell_{i}^{2}-\sum_{j=1}^{\ell_{1}} k_{j}^{2} \\
& U(r)= \begin{cases}r^{2} / N^{2} & \text { for } \mathrm{SU}(N), \\
0 & \text { for } \mathrm{U}(N), \\
r / N & \text { for } \mathrm{SO}(N), \\
-r / N & \text { for } \operatorname{Sp}(N),\end{cases}
\end{aligned}
$$

with $\ell_{i}\left(k_{j}\right)$ denoting the row (column) lengths of the Young diagram corresponding to the representation $R$, and $r=\sum_{i=1}^{k_{1}} \ell_{i}$ is the number of cells in the Young diagram. The dimension of a representation $R$ of $\mathrm{SU}(N)$ whose Young diagram has a finite number of cells $r$ in the limit $N \rightarrow \infty$ can be written [6]

$$
\operatorname{dim} R=\frac{N^{r}}{r !} \chi_{R}\left(\Omega_{r}\right)
$$

where

$$
\Omega_{r}=\sum_{\sigma \in S_{r}} N^{c(\sigma)-r} \sigma \quad \text { for } \mathrm{SU}(N)
$$

with $c(\sigma)$ the number of cycles in the permutation $\sigma$. The dimension of a "composite" representation [5] $R \bar{S}$ of $\mathrm{SU}(N)$, where $R$ and $S$ each have Young diagrams with a finite number of cells, almost factorizes

$$
\operatorname{dim}(R \bar{S})=\operatorname{dim}(R) \operatorname{dim}(\bar{S})\left[1+O\left(\frac{1}{N^{2}}\right)\right]
$$

The dimensions of tensor representations of $\mathrm{SO}(N)$ and $\operatorname{Sp}(N)$ are also given by eq. (3.3), but the operator $\Omega_{r}$ has sub-leading terms [7-9]

$$
\Omega_{r}=\sum_{\sigma \in S_{r}} N^{c(\sigma)-r}\left[1+O\left(\frac{1}{N}\right)\right] \sigma \quad \text { for } \operatorname{SO}(N) \text { and } \operatorname{Sp}(N) .
$$

In the string theory, the first term in the quadratic Casimir (3.1) weights each map with the exponential of the worldsheet area, the Nambu-Goto action. The 
second term corresponds to the presence of an arbitrary number of simple branch points [5]. Both these terms are universal among the different gauge groups. The third term in the Casimir corresponds to other features [5, 7] of the string maps (infinitesimal handles, cross-caps, and orientation-preserving tubes [OPT]) which differ among the gauge groups. In addition, orientation-reversing tubes (ORT) arise from sub-leading terms in the quadratic Casimir of composite representations of $\mathrm{SU}(N)$. The $\operatorname{dim} R$ term also gives rise to various features of the string maps. The $\Omega_{r}$ operator (3.4) is called a twist-point operator, each of its terms permuting the sheets in the manner of a multiple branch point [6]; its leading term is universal among the gauge groups. The sub-leading terms in (3.5) may be attributed to the presence of infinitesimal ORTs connecting cycles of equal length [6]; we call these "twist-point tubes." The sub-leading terms in (3.6) also give rise to twist-point tubes, and to infinitesimal cross-caps on cycles of even length [8].

\begin{tabular}{|l|r|r|}
\hline GAUGE GROUP & $\mathrm{SU}(N)$ & $\mathrm{SO}(N) / \mathrm{Sp}(N)$ \\
\hline Nambu-Goto Action & $\exp (-r A / 2)$ & $\exp (-r A / 2)$ \\
\hline AREA-DEPENDENT FEATURES & $-A / N$ & $-A / N$ \\
\hline Simple Branch Points & $A / N^{2}$ & \\
\hline Orientation Preserving Tubes (OPT) & $-A / N^{2}$ & $\pm / 2 N^{2}$ \\
\hline Orientation Reversing Tubes (ORT) & & $1 / N^{r-c(\sigma)}$ \\
\hline Handles & & $-1 / N^{2}$ \\
\hline Crosscaps & $1 / N^{r-c(\sigma)}$ & $\mp 1 / N$ \\
\hline AREA-INDEPENDENT FEATURES & $-1 / N^{2}$ & \\
\hline Multiple Branch (Twist) Points & & \\
\hline ORTs between equal length cycles & & \\
\hline Tubes between equal length cycles & & \\
\hline Crosscaps on even length cycles & & \\
\hline
\end{tabular}

TABLE I: Weights assigned to string map features

The weights assigned to each of the string features discussed above are sum- 
marized in Table I. ${ }^{*}$ Those features that may appear anywhere on the target space (such as simple branch points) are weighted with a factor of area, so we call them "area-dependent." Features that are immobile (such as twist points) we call "areaindependent."

Another difference between the gauge groups is that the worldsheets of the $\mathrm{SU}(N)$ and $\mathrm{U}(N)$ string theories have a definite orientation. Thus, the map from a worldsheet to an oriented target space $\mathcal{S}$ is at any point either orientationpreserving or orientation-reversing [5]. A given worldsheet can have both orientationpreserving and orientation-reversing components; one thus refers to the chiralities of the various components of the worldsheet. On the other hand, worldsheets of the $\operatorname{SO}(N)$ and $\operatorname{Sp}(N)$ string theories need not be orientable [7].

In summary, then, the string theories corresponding to $\mathrm{SU}(N), \mathrm{U}(N), \mathrm{SO}(N)$, and $\operatorname{Sp}(N)$ Yang-Mills theory have in common the same worldsheet branch- and twist-point structures. They differ in terms of possible insertions of infinitesimal handles, tubes, and crosscaps (both area-dependent and -independent), and also in that $\mathrm{SU}(N)$ and $\mathrm{U}(N)$ worldsheets are oriented whereas $\mathrm{SO}(N)$ and $\operatorname{Sp}(N)$ worldsheets are not. To understand the large $N$ universality of Wilson loops, we must explain how the differences between the theories (as shown in Table I) can lead to the same values for VEVs.

The unnormalized VEV $W_{\kappa}(2.2)$ has a natural stringy interpretation [6,8-10]. Each term in the $1 / N$ expansion of $W_{\kappa}$ corresponds to a map from a worldsheet with boundary to $\mathcal{S}$, where the worldsheet boundary is mapped to the Wilson loop. If the worldsheet has Euler characteristic $\mathcal{E}$, then its contribution to $W_{\kappa}$ is proportional to $N^{\mathcal{E}}$. The conjugacy class $\kappa$ determines the boundary conditions on the maps. If the conjugacy class $\kappa$ has $c$ cycles, with cycle lengths $n_{i}(i=1, \ldots, c)$, then the worldsheet has $c$ boundary components $B_{i}$, and each of the curves $B_{i}$ is mapped with degree $n_{i}$ to the Wilson loop (i.e., traversing the curve $B_{i}$ once,

\footnotetext{
* The string version of the $U(N)$ gauge theory is very similar to that of $S U(N)$, differing only in the absence of area-dependent tubes and handles.
} 
one circles the Wilson loop $n_{i}$ times). Maps that contribute to $W_{\kappa}$ can be from surfaces with arbitrarily large Euler characteristic, and therefore contribute with arbitrarily large power of $N$. However, these maps correspond to disconnected worldsheets. Normalizing the Wilson loop expectation value by dividing by the Yang-Mills partition function,

$$
\widehat{W}_{\kappa}=W_{\kappa} / Z
$$

removes the disconnected "vacuum graphs," and results in a quantity with a large $N$ limit that we will show is universal.

Having described the possible connected string maps that contribute to the normalized Wilson loop VEV, we now analyze those which dominate in leading order in $1 / N$. We see immediately that some of the features discussed above are irrelevant in leading order. Any $\mathrm{SU}(N)$ worldsheet with an infinitesimal handle will be suppressed by $1 / N^{2}$ relative to the same worldsheet with the handle removed, and so is negligible. Likewise, any $\mathrm{SO}(N)$ or $\mathrm{Sp}(N)$ worldsheet with an infinitesimal crosscap is down by $1 / N$ relative to the surface without it. (Since the weights of $\mathrm{SO}(N)$ and $\operatorname{Sp}(N)$ maps differ only in the sign of the crosscaps, it immediately follows that $\mathrm{SO}(N)$ and $\mathrm{Sp}(N)$ Wilson loop VEVs are equal to leading order in $1 / N$.) Also, in general, $\mathrm{SO}(N)$ and $\mathrm{Sp}(N)$ Wilson loops can have contributions from nonorientable worldsheets, but such worldsheets are always suppressed by at least a factor of $1 / N$ relative to a contributing orientable worldsheet.

Since infinitesimal tubes are associated with a factor of $1 / N^{2}$, one might suppose that they would also be irrelevant to leading order in $1 / N$. This is not the case. Any infinitesimal tube whose removal leaves a map that contributes to the normalized Wilson loop VEV can indeed be ignored. It is possible, however, that the removal of a tube could result in a surface with a disconnected vacuum part; such a surface does not contribute to the normalized VEV. If the contribution of this disconnected worldsheet is of higher order in $N$ than the connected worldsheets, then the insertion of a tube, which reduces the $N$ dependence, can result in a surface that contributes to the same order as the other connected worldsheets. 
Thus, to leading order in $1 / N$, we may have to include worldsheets with infinitesimal tubes, but only if the removal of any tube results in a disconnected vacuum piece. Therefore, one concludes that the tube structure of the worldsheets that contribute to leading order in $1 / N$ to the VEV must be tree-like, i.e., not have any closed loops.

Clearly, the set of worldsheet maps with only simple branch points and twist points will be equivalent in all the string theories, irrespective of the gauge group. It is far from obvious, however, that maps that include infinitesimal tubes will be identical, since the tube structures differ between the groups. We will now show that, in fact, the set of worldsheet maps is identical for all the groups. We do this by constructing an isomorphism between these maps: for each worldsheet of $\mathrm{SO}(N) / \mathrm{Sp}(N)$ that contributes to leading order in $1 / N$, we will construct the corresponding worldsheet of $\mathrm{SU}(N)$, and vice versa.

Let us begin with the normalized $\mathrm{VEV} \widehat{W}_{(n)}$ of a non-intersecting Wilson loop on the sphere, where $(n)$ denotes the conjugacy class with a single cycle of length $n$. Consider the most general map that contributes to leading order in $1 / N$ to this Wilson loop in $\mathrm{SO}(N)$ (or $\mathrm{Sp}(N)$ ) string theory. The worldsheet consists of a set of connected components $C_{0}, C_{1}, C_{2}, \cdots, C_{m}$, where $C_{0}$ has the topology of a disk and $C_{1}, C_{2}, \cdots, C_{m}$ are topologically spheres. If any of the components had a higher genus, the Euler characteristic of the worldsheet would be decreased and the map would be sub-leading in the $1 / N$ expansion. The component $C_{0}$ is mapped to the target space sphere $\mathcal{S}$, and its boundary is mapped to the Wilson loop, wrapping around the loop $n$ times. For $n>1$, there will necessarily be branch points in the map $C_{0} \rightarrow \mathcal{S}$. Each of the other components $C_{1}, C_{2}, \cdots, C_{m}$ is a covering of the sphere $\mathcal{S}$ with arbitrary degree $n_{i}$ and branching number $2 n_{i}-2$. The branch points on $C_{0}, \cdots, C_{m}$ can be either simple branch points associated with $T(R)$ in the Casimir, or multiple branch points associated with one of the twist points on $\mathcal{S}$. While the branching structure of any component $C_{i}$ may be very complicated, all of the groups have exactly the same set of branched covers with exactly the same weights. The different components $C_{i}$ are connected to each other in a tree-like 
structure by twist-point tubes (the only kind of tubes in $\mathrm{SO}(N)$ ). In particular, any cycle of sheets at a twist point can be connected to an equal length cycle of sheets on another component. Since there can be no closed loops in the structure, exactly $m$ tubes connect the $m+1$ components. The Euler characteristic of the connected surface is thus $1+2 m-2 m=1$, showing it to have the topology of a disk. Thus, the Wilson loop expectation value $\widehat{W}_{(n)}$ is proportional to $N$ in leading order.

We now describe the $\mathrm{SU}(N)$ map that corresponds to the one just described for $\mathrm{SO}(N) / \mathrm{Sp}(N)$. It has of course the same worldsheet, but with a chirality assigned to each of the components $C_{i}$. The conjugacy class $(n)$ of the Wilson loop is a linear combination of the products of either the fundamental representation $\square$ of $\mathrm{SU}(N)$ or of its conjugate $\overline{\bar{a}}$; this determines the chirality of the Wilson loop itself. The boundary of $C_{0}$ is mapped to the Wilson loop, and the region of $C_{0}$ bordering the boundary will be mapped either to one side of the Wilson loop or to the other. The chirality of $C_{0}$ is fixed by the chirality of the Wilson loop, and depends on which side of the Wilson loop it approaches. What are the chiralities of the other components $C_{i}$ ? In $\mathrm{SU}(N)$, twist points tubes only connect sheets of opposite chirality, so any component $C_{i}$ to which $C_{0}$ is directly connected must have chirality opposite that of $C_{0}$. Any components connected directly to these components have the same chirality as $C_{0}$, and so forth. Thus, the chirality of each of the components of the $\mathrm{SU}(N)$ worldsheet is uniquely fixed by the chirality of the Wilson loop and the connectivity of the structure. Since there are no closed loops in the tube structure, no ambiguity can result from this prescription. Thus, to each $\mathrm{SO}(N) / \mathrm{Sp}(N)$ map, there corresponds a well-defined $\mathrm{SU}(N)$ map with the same weight (since the twist point tubes have the same sign and weight for $\mathrm{SU}(N)$ and $\mathrm{SO}(N) / \mathrm{Sp}(N))$.

To demonstrate the isomorphism in the other direction, take an $\mathrm{SU}(N)$ worldsheet and erase the chiralities of each of the components to obtain the corresponding $\mathrm{SO}(N) / \mathrm{Sp}(N)$ worldsheet. 
It might be thought that we have neglected a whole set of maps in the $\mathrm{SU}(N)$ string theory, namely those containing infinitesimal area-dependent tubes. (There are no area-dependent tubes in $\mathrm{U}(N)$.) In fact, to leading order in $1 / N$, the contributions of area-dependent OPTs and ORTs (tubes that connect sheets of the same or opposite chirality respectively) to the Wilson loop VEV exactly cancel [18]. To see this, consider some worldsheet connected by an OPT to an otherwise disconnected vacuum piece $V$. (This is the only type of worldsheet allowed in leading order.) Then consider the same worldsheet connected by an ORT to $\bar{V}$, where $\bar{V}$ is obtained from $V$ by reversing the chiralities of each of its components. The weights of these two maps are equal up to an overall sign, and so they cancel. Thus, only (area-independent) twist-point tubes contribute in $\mathrm{SU}(N)$ to leading order in $1 / N$.

By exhibiting the large $N$ isomorphism of the string maps in the $\mathrm{SU}(N), \mathrm{U}(N)$, $\mathrm{SO}(N)$, and $\operatorname{Sp}(N)$ string theories, we have just demonstrated the universality of normalized Wilson loops $\widehat{W}_{(n)}$ for conjugacy classes with a single cycle on $S^{2}$. Next, we consider a normalized Wilson loop VEV $\widehat{W}_{\kappa}$, where the conjugacy class $\kappa$ has more than one cycle, $\kappa=\left(n_{1}\right)\left(n_{2}\right) \ldots\left(n_{c}\right)$. To leading order in $1 / N$, it is clear that the maps that contribute to this Wilson loop expectation value will simply be disconnected products of the maps that contribute to each of the cycles $\left(n_{i}\right)$. (Dividing by $Z$ removes disconnected vacuum components for $W_{\kappa}$, but the maps may still be disconnected.) Any connected map will be suppressed by at least one power of $1 / N$. Thus, to leading order in $1 / N$,

$$
\widehat{W}_{\kappa}=\prod_{i=1}^{c} \widehat{W}_{\left(n_{i}\right)} \underset{N \rightarrow \infty}{\longrightarrow} O\left(N^{c}\right)
$$

so from the previous result, it follows that $\widehat{W}_{\kappa}$ is identical for each of the groups in the large $N$ limit. The same argument shows that the expectation value of an arbitrary number of non-intersecting homologically trivial Wilson loops on a sphere is universal. This is because the expectation value is the product of the expectation values for each of the separate Wilson loops. 
The proof is easily extended to homologically trivial Wilson loops on the torus and on higher genus orientable target spaces. In these cases, the only string maps that contribute to leading order in $1 / N$ are those with a single component $C_{0}$ with the topology of the disk; maps with any twist-point tubes are easily seen to be sub-leading. This greatly reduced set of contributing worldsheets leads to much simpler expressions for the universal VEVs for homologically trivial Wilson loops on any orientable surface of genus $h \geq 1$; for example,

$$
\begin{array}{ll}
\widehat{W}_{(1)} \underset{N \rightarrow \infty}{\longrightarrow} N \mathrm{e}^{-\frac{1}{2} A_{1}}, & \\
\widehat{W}_{(2)} \underset{N \rightarrow \infty}{\longrightarrow} N\left(1-A_{1}\right) \mathrm{e}^{-A_{1}}, & h \geq 1 \\
\widehat{W}_{(3)} \underset{N \rightarrow \infty}{\longrightarrow} N\left(1-3 A_{1}+\frac{3}{2} A_{1}^{2}\right) \mathrm{e}^{-\frac{3}{2} A_{1}} . &
\end{array}
$$

Wilson loops also have universal leading-order expectation values on nonorientable surfaces. For the homologically trivial Wilson loop $\widehat{W}_{(1)}$ on $R P^{2}$, the worldsheets that contribute to leading order in $1 / N$ will have the same structure as those for $S^{2}$. Each worldsheet will consist of connected components $C_{0}, C_{1}, \cdots, C_{m}$, where $C_{0}$ has the topology of a disk and the others that of a sphere. The maps from $C_{i}$ to $R P^{2}$ must all have even degree. The $\mathrm{SO}(N)$ and $\operatorname{Sp}(N)$ string theories also allow maps of odd degree, corresponding to nonorientable worldsheets, but these will all be higher order in $1 / N$ relative to the orientable worldsheet contributions since they involve cross-cap insertions. Thus, there is again an isomorphism of maps, and an equality of Wilson loop expectation values. For example, the normalized Wilson loop in the fundamental representation has the universal leading order expectation value

$$
\widehat{W}_{(1)} \underset{N \rightarrow \infty}{\longrightarrow} N\left[\mathrm{e}^{-\frac{1}{2} A_{1}}+\mathrm{e}^{-\frac{1}{2} A_{1}-A_{2}}+\left(\frac{1}{2} A_{1}^{2}-A_{1}-1\right) \mathrm{e}^{-\frac{3}{2} A_{1}-A_{2}}+\cdots\right] .
$$

For nonorientable surfaces of higher genus, the only string maps that contribute in leading order in $1 / N$ to homologically trivial Wilson loops are those with a single component $C_{0}$ with the topology of the disk. 
Homologically non-trivial Wilson loops are also universal in the large $N$ limit. For example, the homologically non-trivial Wilson loop on the torus with $\kappa=(1, \overline{1})$ (which reduces to $\kappa=\left(1^{2}\right)$ for $\mathrm{SO}(N) / \mathrm{Sp}(N)$ ) has the universal large $N$ normalized $\mathrm{VEV}$

$$
\widehat{W}_{(1, \overline{1})} \underset{N \rightarrow \infty}{\longrightarrow} 2\left(\mathrm{e}^{-\frac{1}{2} A}+\mathrm{e}^{-A}+\mathrm{e}^{\frac{3}{2} A}+\mathrm{e}^{-2 A}+\cdots\right)=\frac{2}{\mathrm{e}^{\frac{1}{2} A}-1}
$$

where $A$ is the dimensionless area of the torus. The terms in the sum correspond to multiple covers of the cylinder by the cylinder. We anticipate no obstacles to extending the string proof of universality to the general case of homologically non-trivial Wilson loops on higher genus surfaces.

\section{Phase transitions}

Large $N$ Yang-Mills theories on the two-sphere have a strong- and weakcoupling phase separated by a third-order phase transition [12, 11]. Although the most direct way to understand this is in terms of the related matrix model, it is interesting to examine this phase transition in string-theoretic terms [18]. Toward this end, we call attention to the behavior of the string sums contributing to Wilson loops as one approaches the critical point.

Restricting our attention to $\mathrm{SU}(N)$ Yang-Mills theory in this section, we recall that the string theory of $\mathrm{QCD}_{2}$ is formulated as a strong-coupling expansion that splits into two nearly independent chiral sectors [5]. (This split, and the individual chiral theories themselves, may not be sensible for all coupling.) We can test the convergence of the contributions to the individual chiral sectors on $S^{2}$ by studying the expansion for the Wilson loop directly, analogous to the calculation carried out by Taylor for the partition function [18].

We may pick out the contributions to the Wilson loop VEV in the fundamental representation on the sphere (2.3) due to maps from a single chiral sector containing only mobile branch points. This subset of string maps corresponds to the "B1" 
string theory of ref. [18]. In the large $N$ limit, only simply-connected maps in the B1 theory contribute, and for a single chiral sector, these maps possess mobile branch points in area $A_{1}$ only (cf. eq. (2.3))

$$
\begin{aligned}
\frac{\widehat{W}_{(1)}\left(A_{1}, A_{2}\right)}{N} & \underset{N \rightarrow \infty}{\longrightarrow} \mathrm{e}^{-\frac{1}{2} A_{1}}+\frac{1}{2} A_{1}^{2} \mathrm{e}^{-A_{1}-\frac{1}{2} A_{2}}+\frac{1}{2} A_{1}^{4} \mathrm{e}^{-\frac{3}{2} A_{1}-A_{2}}+\cdots \\
& =\sum_{n=1}^{\infty} \Gamma_{n} \frac{A_{1}^{2 n-2}}{(2 n-2) !} \mathrm{e}^{-\frac{1}{2} n A_{1}-\frac{1}{2}(n-1) A_{2}}
\end{aligned}
$$

The coefficient $\Gamma_{n}$ counts the number of distinct string maps from surfaces consisting of $n-1$ spheres and 1 disk simply-connected by $2 n-2$ branch points to the target space disk. A closely related combinatorial problem $[18,17]$ is the number of distinct string maps from $n$ spheres simply-connected by $2 n-2$ branch points to the sphere

$$
G_{n}=n^{n-3} \frac{(2 n-2) !}{n !}
$$

These coefficients are related by $\Gamma_{n}=n G_{n}$, since the disk is distinct from the rest of the worldsheet (spheres) and thus the overall symmetry factor is reduced.

Equation (4.1) converges for $A_{1}$ sufficiently large, regardless of the value of $A_{2}$. Taking $A_{2}$ to zero in (4.1), the resulting expression is convergent for $A_{1}$ greater than a certain area $A_{c}$,

$$
\frac{\widehat{W}_{(1)}\left(A_{1}, 0\right)}{N} \underset{N \rightarrow \infty}{\longrightarrow} \sum_{n=1}^{\infty} \frac{n^{n-2}}{n !} A_{1}^{2 n-2} \mathrm{e}^{-\frac{1}{2} n A_{1}}
$$

This expression has identical convergence properties to that of the B1 free energy on a sphere of area $A_{1}$, and converges outside the region $0.73 \lesssim A_{1} \lesssim 11.9$. This divergence below $A_{1} \approx 11.9$ may be related to the well-known third-order phase transition. The relation between the non-analytic behavior of quantities such as the free energy and that of Wilson loop VEVs is poorly understood. Above we have shown that the behavior of part of the Wilson loop VEV in two dimensions mimics the behavior of the free energy at large $N$. 


\section{Conclusions}

Wilson loops on an arbitrary surface have universal large $N$ VEVs for all gauge groups. This fact is unexpected and obscure in the gauge theory expressions for these observables, but is easily understood from the underlying string description. In this paper, we have used the string description to present a general proof of the large $N$ universality of Wilson loops. Understanding universality in the strongcoupling limit is thus a significant application of the string approach.

The analysis and results of this paper complement those of ref. [11], in which universality was first found using large $N$ matrix model techniques. Furthermore, we have also shown that the chiral part of the large $N$ expression for an $\mathrm{SU}(N)$ Wilson loop on the sphere fails to converge below a certain coupling, perhaps indicating the presence of a phase transition as suggested in [18].

These results suggest the existence of an underlying universal theory on the worldsheet to leading order in $1 / N$, such as that proposed in ref. [19]. The $O(1 / N)$ corrections to these theories are not universal $[7,8]$. Corrections to the $\mathrm{SU}(N)$ partition function in the weak coupling phase have been worked out in ref. [20]; further developments will be presented in ref. [21].

\section{Acknowledgements}

We thank K. Bardakci, H. Riggs, and W. Taylor for comments. 


\section{REFERENCES}

1. A. Migdal, Sov. Phys. JETP 42 (1975) 413.

2. N. Bralic, Phys. Rev. D22 (1980) 3090;

V. Kazakov and I. Kostov, Nucl. Phys. B176 (1980) 199.

3. B. Rusakov, Mod. Phys. Lett. A5 (1990) 693;

D. Fine, Commun. Math. Phys. 134 (1990) 273; 140 (1991) 321;

E. Witten, Commun. Math. Phys. 141 (1991) 153;

M. Blau and G. Thompson, Int. Jour. Mod. Phys. A7 (1992) 3781.

4. G. 't Hooft, Nucl. Phys. B75 (1974) 461;

W. Bardeen, I. Bars, A. Hanson, and R. Peccei, Phys. Rev. D13 (1976) 2364.

5. D. Gross, Nucl. Phys. B400 (1993) 161;

J. Minahan, Phys. Rev. D47 (1993) 3430;

D. Gross and W. Taylor, Nucl. Phys. B400 (1993) 181.

6. D. Gross and W. Taylor, Nucl. Phys. B403 (1993) 395.

7. S. Naculich, H. Riggs, and H. Schnitzer, Mod. Phys. Lett. A8 (1993) 2223;

Phys. Lett. B466 (1993) 466.

8. S. Ramgoolam, Nucl. Phys. B418 (1994) 30.

9. S. Naculich, H. Riggs, and H. Schnitzer, hep-th/9406100, Int. Jour. Mod. Phys. A, in press;

S. Naculich and H. Riggs, hep-th/9411143, Phys. Rev. D, in press.

10. S. Ramgoolam, hep-th/9412110.

11. M. Crescimanno and H. Schnitzer, hep-th/9501099.

12. M. Douglas and V. Kazakov, Phys. Lett. B319 (1993) 219.

13. D. Boulatov, Mod. Phys. Lett. A9 (1994) 365.

14. B. Rusakov, Phys. Lett. B303 (1993) 95. 
15. J. Minahan and A. Polychronakos, Nucl. Phys. B422 (1994) 172.

16. J. Daul and V. Kazakov, Phys. Lett. B355 (1994) 371;

B. Rusakov, Phys. Lett. B329 (1994) 338.

17. M. Crescimanno and W. Taylor, hep-th/9408115.

18. W. Taylor, hep-th/9404175.

19. P. Horava, hep-th/9311156;

S. Cordes, G. Moore, and S. Ramgoolam, hep-th/9402107.

20. D. Gross and A. Matytsin, Nucl. Phys. B429 (1994) 50; hep-th/9410054.

21. M. Crescimanno, S. Naculich, and H. Schnitzer, in preparation. 